\title{
Análisis de las megatendencias de educación superior
}

\author{
Analysis of higher education's megatrends
}

\section{INTRODUCCIÓN}

Alvin Toffler, en su libro El Shock del futuro en los años 70 (1), sostenía que el mundo estaba enfrentado al inicio de fuertes cambios en su sustrato tecnológico y social, y que se estaban gestando cambios significativos en las personas, en las organizaciones y en los grupos sociales; cuyas dinámicas imponían nuevos desafíos, especialmente a las instituciones de educación, y en particular a las universidades como las instituciones tradicionales generadoras y transmisoras de conocimiento, que están en el centro mismo de los "shocks", puesto que son los instrumentos y las palancas en el camino hacia la nueva sociedad del conocimiento que se está generando a escala global y que está rediseñando el mapa político, comercial y productivo (2).

Este tercer "shock" que enfrentan las sociedades, golpea particularmente a los sistemas universitarios y está promoviendo en América Latina una metamorfosis de los sistemas universitarios, una mutación expresada en la tercera reforma de la educación superior, la cual es, sin duda, la más compleja, generalizada y riesgosa por la dimensión del impacto, la cantidad de variables en juego y la vinculación con tantas áreas de la sociedad (2).

\section{La Primera Reforma: La autonomía y el cogobierno}

América Latina tuvo su Primera Reforma sobre

la educación superior hacia comienzos del siglo $\mathrm{XX}$, como respuesta a las nuevas demandas de las capas medias urbanas, a los requerimientos que implicaba la conformación de los estados modernos, a la industrialización por sustitución de importaciones y a la vigorosa urbanización, todo lo cual requirió la democratización y la expansión de las universidades y una nueva orientación hacia la formación de los profesionales (2).

Este fue el detonante de la Primera Reforma Universitaria en América Latina, marcada por la Reforma de Córdoba en 1918, que se fue expandiendo e instalando por toda la región y que, al promover la autonomía y el cogobierno universitario, contribuyó decididamente a la expansión de la

1 Ingeniero Electricista; MBA por la Universidad de Québec en Montreal; estudios de doctorado en Administración y Economía de Negocios en la Universidad de Sevilla. Diplomas del Programa de Alta Dirección y del Programa para Presidentes y Gerentes en la Universidad de Piura; Diploma del Management Innovation Program del Babson College, USA; Diploma del Programa Avanzado de Dirección de Empresas de la Universidad ESAN. Socio fundador y presidente de la Organización Educativa Continental. 
cobertura de las universidades públicas, superando los modelos de elite y democratizando el acceso a la educación superior a nuevos contingentes urbanos que gracias a la formación profesional accedieron a una significativa movilidad social. Tal modelo monopólico educativo universitario, público, laico, gratuito, cogobernado y autonomista, tuvo larga y destacada vigencia y se fue imponiendo progresivamente en cada uno de los países de la región hasta inicios de los setenta, cuando la crisis de los modelos económicos golpearon las puertas de las instituciones de educación superior y sentaron las bases de una nueva transformación de la educación superior (2).

\section{La Segunda Reforma: La mercantilización y diferenciación}

Un nuevo y radical movimiento estudiantil latinoamericano mostró claramente que las universidades, tal como estaban estructuradas no respondían a los nuevos escenarios políticos y económicos ni a las nuevas demandas sociales. El movimiento estudiantil expresaba tanto la necesidad de promover renovaciones en los sistemas universitarios, como las urgencias de crecientes masas de bachilleres, que presionaban para ampliar la cobertura de la educación superior (2).

Este proceso constituyó el centro de la Segunda Reforma de la educación superior en el continente desde los ochenta, y que promovió un incremento de la cobertura en el marco de una fuerte diferenciación de las instituciones y de la calidad de los servicios educativos, y que terminó conformando un nuevo modelo universitario de carácter dual. Se caracterizó por la instauración de un complejo modelo binario público y privado, de alta y baja calidad, universitario y no universitario, como resultado del contexto mercantil y heterogéneo, y que promovió modelos de calidad, de precios, de financiamiento diferenciados. Ello facilitó la significativa expansión del sector privado, medido en términos de cobertura, matrícula e instituciones, sobre la base, en general, de un pequeño sector de calidad y otro mayor orientado a la absorción de la demanda insatisfecha y con menores exigencias de calidad (2).

Adicionalmente, la crisis de esta etapa de la educación superior en América Latina se produjo junto a un incremento significativo de la demanda estudiantil y del tercer "shock" sobre las sociedades a escala global, como analizara Toffler, marcado por la creciente renovación de saberes en el marco de las sociedades del conocimiento, del desarrollo de nuevas tecnologías de información y comunicación, de la globalización económica y de la creciente internacionalización de la educación superior (2).

Claudio Rama sostiene que todos ellos son los fenómenos que coadyuvan a sentar las bases del inicio de la Tercera Reforma de la educación superior en el continente (3).

\section{La Tercera Reforma: Masificación e internacionalización}

Un verdadero "shock" sufren hoy en América Latina los sistemas de educación superior a causa de la internacionalización de este nivel de educación, las nuevas tecnologías de comunicación e información, las nuevas demandas de acceso de la población, incluyendo sectores fuertemente marginados con anterioridad como los grupos indígenas u otras minorías como las personas con discapacidad o los migrantes, y la presencia creciente de sociedades del conocimiento que promueven la educación a lo largo de la vida, la mercantilización del conocimiento y la renovación permanente de los saberes. Directa e indirectamente la internaciona-lización en curso está promoviendo la movilidad estudiantil como parte constitutiva de las nuevas dinámicas de aprendizaje en la sociedad del saber, el establecimiento de estándares internacionales de calidad sobre la educación superior, la presión hacia nuevas pertinencias globales y 
locales y la vinculación de los ciclos y procesos educativos a escala global. El impacto de las nuevas tecnologías por su parte, está contribuyendo a la globalización de la educación, permitiendo acortar las distancias, expandir la educación transfronteriza y las modalidades de educación en red (2).

Así, globalización y sociedad del saber constituyen dos grandes motores que están impulsando la masificación de la educación superior en América Latina, la cual se expresa tanto a través de los niveles de competencia en los mercados laborales como de la disposición de los hogares a sacrificar rentas y tiempo para capacitarse (2).

\section{La tendencia a la masificación y la feminización de la cobertura}

El siglo XX ha sido el siglo de la democratización política de las sociedades en latino américa y el comienzo de la democratización en los accesos a los diversos servicios sociales. El resultado de

Tabla $N^{\circ} 1$ : Características de las fases de la educación superior

\begin{tabular}{|c|c|c|c|}
\hline $\begin{array}{l}\text { Modelo Educación } \\
\text { Superior }\end{array}$ & Modelo político & Objetivos políticos & Instrumento \\
\hline $\begin{array}{l}\text { Primera Reforma: } \\
\text { Autonomía y cogobierno. } \\
\text { Modelo monopólico } \\
\text { público. }\end{array}$ & $\begin{array}{l}\text { Lógica pública. } \\
\text { Lucha por la autonomía. }\end{array}$ & $\begin{array}{l}\text { Búsqueda de fondos. } \\
\text { Estado educador. }\end{array}$ & $\begin{array}{l}\text { Luchas políticas, alianzas } \\
\text { con estudiantes y partidos. }\end{array}$ \\
\hline $\begin{array}{l}\text { Segunda Reforma: } \\
\text { Mercantilización. } \\
\text { Modelo dual público - } \\
\text { privado. }\end{array}$ & $\begin{array}{l}\text { Diversificación } \\
\text { Lógica privada. } \\
\text { Lucha por libertad de } \\
\text { mercado. } \\
\text { Restricciones a la } \\
\text { educación pública. }\end{array}$ & $\begin{array}{l}\text { Competencia por los } \\
\text { estudiantes. } \\
\text { Libertad de enseñanza. }\end{array}$ & $\begin{array}{l}\text { Competitividad basada } \\
\text { en la publicidad y en } \\
\text { diferenciaciones de } \\
\text { calidad-precios. }\end{array}$ \\
\hline $\begin{array}{l}\text { Tercera Reforma: } \\
\text { Internacionalización. } \\
\text { Modelo trinario (público - } \\
\text { privado -internacional). }\end{array}$ & $\begin{array}{l}\text { Lógica nacional defensiva. } \\
\text { Sistemas de aseguramiento } \\
\text { de la calidad. } \\
\text { Asociaciones rectorales. } \\
\text { Nuevo rol del Estado. }\end{array}$ & $\begin{array}{l}\text { Búsqueda de regulación } \\
\text { pública nacionales e } \\
\text { internacionales. } \\
\text { Incremento de cobertura. } \\
\text { La educación como un } \\
\text { bien público internacional. }\end{array}$ & $\begin{array}{l}\text { Alianzas internacionales. } \\
\text { Educación transfronteriza. } \\
\text { Posgrados. } \\
\text { Nueva competencia } \\
\text { internacional. }\end{array}$ \\
\hline $\begin{array}{l}\text { Modelo Educación } \\
\text { Superior }\end{array}$ & Modelo libertad & Concepto político & Instrumento \\
\hline $\begin{array}{l}\text { Reforma: } \\
\text { Autonomía. } \\
\text { Monopolios públicos. }\end{array}$ & Libertad de cátedra. & Bien estatal. & Autonomía. \\
\hline $\begin{array}{l}\text { Reforma: } \\
\text { Mercantilización. } \\
\text { Modelo binario. }\end{array}$ & Libertad de enseñanza. & Bien libre nacional. & Libertad de gestión privada. \\
\hline $\begin{array}{l}\text { Reforma: } \\
\text { Internacionalización. } \\
\text { Modelo tripartito. }\end{array}$ & $\begin{array}{l}\text { Libertad de las } \\
\text { Diversidades. }\end{array}$ & $\begin{array}{l}\text { Bien público. } \\
\text { Consejos de rectores y } \\
\text { organismos públicos. }\end{array}$ & $\begin{array}{l}\text { Regulaciones nacionales, } \\
\text { regionales, internacionales. } \\
\text { Creación de sistemas } \\
\text { nacionales de educación } \\
\text { superior. }\end{array}$ \\
\hline
\end{tabular}


ese proceso ha sido la masificación de la educación básica y de la educación media, que ha sentado las bases de la creciente masificación de la educación superior que será una de las tendencias del siglo XXI (3). La masificación en este último nivel educativo ha sido el resultado de la nueva estrategia de sobrevivencia de los hogares latinoamericanos, dado los menores niveles de desempleo y los mayores ingresos salariales que comparativamente reciben los profesionales universitarios en los mercados laborales.

Los hogares latinoamericanos han seleccionado históricamente los mecanismos más eficientes para lograr la movilidad social. En los cincuenta en el empleo público cumplió ese rol. En los sesenta tal estrategia de sobrevivencia se expresó en el ingreso de las mujeres en los mercados laborales y el aumento en la cantidad de horas trabajadas por los hombres. Desde los ochenta, la nueva estrategia de sobrevivencia de los hogares ha sido la migración y el aumento de los años de escolarización con una creciente tendencia hacia la educación terciaria (3).

Este proceso ha promovido el incremento de la cobertura en la educación superior, y dadas las restricciones de cupos en la educación pública, la respectiva demanda ha derivado en un incremento de la educación privada y por ende a un creciente sacrificio de rentas de los hogares para cubrir los gastos de las matrículas. Tal proceso de estrategia de sobrevivencia de los hogares expresado en el sacrificio de rentas, es la derivación de las demandas de competencias profesionales en mercados laborales cada vez más complejos, diferenciados y precarios (3).

La tabla $N^{\circ} 3$ muestra la importancia y la dimensión del proceso de masificación de la educación superior en América Latina, se percibe una tasa de crecimiento que a su vez aumenta prácticamente cada cinco años. Ello ha permitido que en apenas 35 años la región haya pasado de una cobertura de apenas $7 \%$ en 1970 a $31,1 \%$ en el 2005 sobre una población de 20 a 24 años.

La figura $N^{\circ} 1$ también muestra el comportamiento del incremento absoluto de la cantidad de estudiantes en la región, de 1,6 millones a 15,7 millones al 2005 .

La demanda por acceso a la educación ha promovido el pasaje de una educación de élites a una educación de masas, y ha

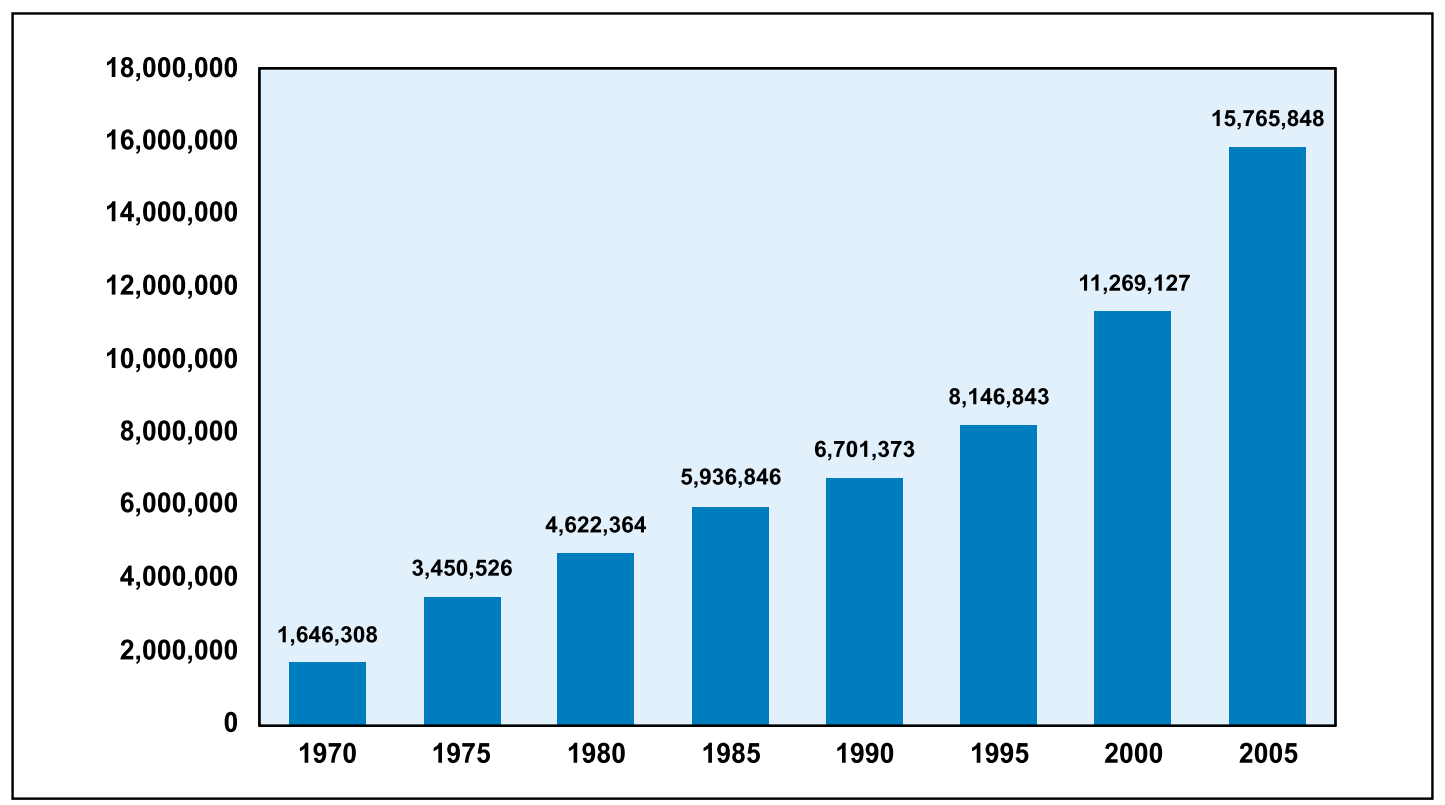

Figura $\mathrm{N}^{\circ} 1$ : Matrícula de educación superior en América Latina: 1970-2005.

Fuente: Estudio de tendencias de educación superior en América Latina, Claudio Rama Vitale. Asamblea Nacional de Rectores, Perú 
Tabla №3: Matrícula de educación superior en América Latina: 1970-2005

\begin{tabular}{llllllllll}
\hline & 1970 & 1975 & 1980 & 1985 & 1990 & 1995 & 2000 & 2005 \\
\hline Matrícula por cada 1 000 habs. & 6,1 & 11,0 & 13,1 & 15,1 & 15,5 & 17,2 & 22,0 & 28,6 \\
Matrícula / Pobl. 20-24 & 7,0 & 12,3 & 14,2 & 18,8 & 16,4 & 18,4 & 23,5 & 31,1 \\
\hline
\end{tabular}

Fuente: Estudio de tendencias de educación superior en América Latina, Claudio Rama Vitale. Asamblea Nacional de Rectores Perú.

incentivado múltiples transformaciones en los sistemas universitarios desarrollando diversos mecanismos para ajustarse a esas transformacionesque promueven las familias y para aprovecharse mercantilmente para cubrir esas necesidades. Esta masificación de la cobertura de la educación superior ha sido uno de los motores de los cambios ya que tales demandas han mostrado que las instituciones tienen grandes dificultades para hacer frente a la masificación por tener estructuras rígidas, por su baja articulación a los otros niveles educativos, por su poca diversificación, por la existencia de programas homogéneos y compartimentados por disciplinas y sin capacidad de responder a la transdisciplinariedad, la educación permanente y la diversidad de nuevos intereses y demandas (3).

\section{Crecimiento del sistema universitario}

Desde 1996 que se ejecutó el primer censo universitario, al 2010 que fue realizado el segundo censo, apreciamos un aumento notable en las instituciones universitarias a nivel nacional.

En el censo universitario 1996 se censaron en el país 57 universidades, casi en igual proporción universidades públicas y privadas (28 públicas y 29 privadas).

En el 2010 fueron censadas 100 universidades, con un considerable crecimiento de las universidades privadas, que ascienden a 65 en total, 1,8 veces más que en 1996 (figura $\mathrm{N}^{\circ} 2$ ).

La población involucrada directamente en el

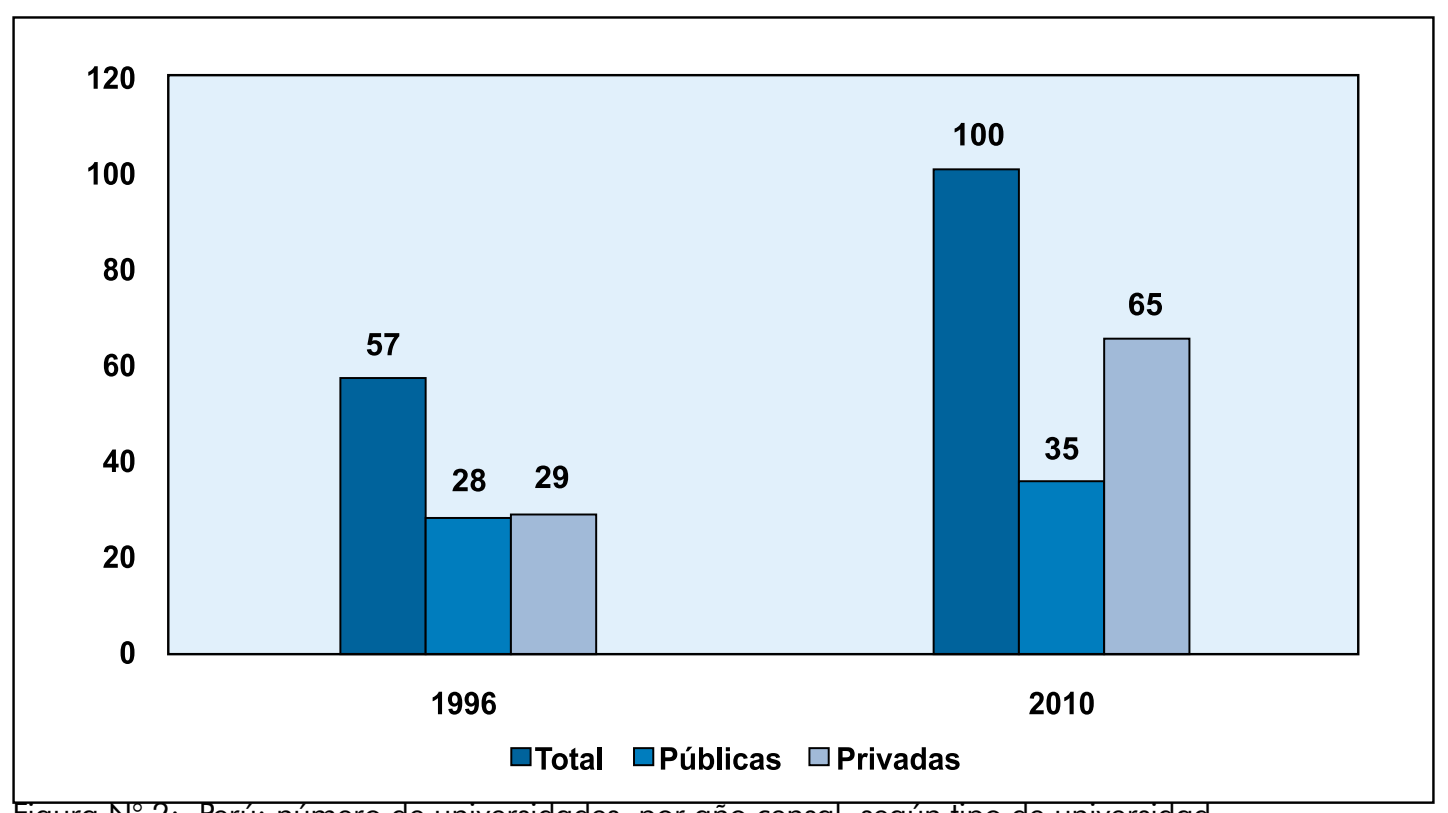

Figura N 2: Perú: número de universidades, por año censal, según tipo de universidad.

Fuente: INEI - I Censo Nacional Universitario, 1996. INEI - II Censo Nacional Universitario, 2010. 
complejo universitario peruano, constituida por estudiantes (de pre y posgrado), docentes $y$ personal administrativo $y$ de servicios, se aproxima al millón de personas. En 14 años (1996 al 2010) se ha incrementado en 2,4 veces, de 389316 a 937430 personas.

Este crecimiento es diferencial entre la gestión pública y privada. Los estudiantes de pregrado son quienes representan el mayor volumen poblacional, ascienden a 782970 en el 2010, el 83,5\% de la población universitaria.

En 1996 los estudiantes de las universidades públicas conformaban la mayor parte $(59,6 \%$ del total), pero la universidad privada, en virtud de su acelerado crecimiento, logra posicionarse en el 2010 como la de mayor población estudiantil $(60,5 \%)(4)$.

En el período en referencia, los estudiantes de posgrado se multiplicaron por 5,2 veces, de 10818 en 1996 a 56358 en el 2010; los docentes universitarios aumentaron de 25795 a 59 085, y los trabajadores administrativos pasaron de 16989 a 39 017. Las tasas de crecimiento en estos dos últimos estamentos fueron de $5,2 \%$ y $6,1 \%$ anual, respectivamente (tabla $\mathrm{N}^{\circ} 4$ ).

\section{Tasa de matrículas en educación superior: Perú 2002-2011}

De acuerdo con los resultados de la Encuesta Nacional de Hogares 2011 , en la tabla $N^{\circ}$ 5 se muestra la tasa neta de matrícula de la población de 17 a 24 años de edad que estudia educación superior, fue de $27,3 \%$, es decir, 27 de cada 100 personas de 17 a 24 años de edad están matriculados en educación superior.

La matrícula a educación superior de la población de 17 a 24 años de edad, según área de residencia es diferenciada. En el área urbana el 31,9\% está matriculado en un centro de educación superior; mientras que en el área rural solo el 12,4\%.

Al comparar con lo registrado en el 2002 se observa que se ha incrementado la tasa de matrícula en este nivel educativo, pero no en la intensidad de los otros niveles de educación. Los menores incrementos se encuentran en el área rural $(4,2 \%)$ y la región Sierra (6,0\%); en cambio, el aumento de la cobertura de la matrícula es mayor en la regiones de la Costa $(6,3 \%)$ y Selva $(7,4 \%)$.

Tabla № 4: Población universitaria, por año censal y tasa de crecimiento anual, según tipo de universidad.

\begin{tabular}{|c|c|c|c|c|c|}
\hline \multirow{2}{*}{$\begin{array}{l}\text { tipo de } \\
\text { universidad }\end{array}$} & \multirow{2}{*}{$\begin{array}{l}\text { Número de } \\
\text { universidad }\end{array}$} & \multicolumn{2}{|c|}{ Alumnos } & \multirow{2}{*}{$\begin{array}{l}\text { Docentes } \\
\text { universitarios }\end{array}$} & \multirow{2}{*}{$\begin{array}{c}\text { Personal administrativo } \\
y \text { de servicio }\end{array}$} \\
\hline & & Pregrado & Posgrado & & \\
\hline \multicolumn{6}{|c|}{ Año 2010} \\
\hline Total & 100 & 782970 & 56358 & 59085 & 39017 \\
\hline Públicas & 35 & 309175 & 24591 & 21434 & 19961 \\
\hline Privadas & 65 & 473795 & 31767 & 37651 & 19056 \\
\hline \multicolumn{6}{|c|}{ Año 1996} \\
\hline Total & 57 & 335714 & 10818 & 25795 & 16989 \\
\hline Públicas & 28 & 199943 & 7109 & 16096 & 11708 \\
\hline Privadas & 29 & 135771 & 3709 & 9699 & 5281 \\
\hline \multicolumn{6}{|c|}{ Tasa de crecimiento anual: Período 1996-2010 } \\
\hline Total & 4,2 & 6,2 & 12,4 & 5,2 & 16,1 \\
\hline Públicas & 1,6 & 3,1 & 9,2 & 1,4 & 3,9 \\
\hline Privadas & 6,0 & 9,3 & 16,5 & 9,1 & 9,6 \\
\hline
\end{tabular}

Fuente: INEI - I Censo Nacional Universitario, 1996. INEI - II Censo Nacional Universitario, 2010. 
Tabla № 5: Perú: tasa de matrícula de la población de 17 a 24 años de edad a educación superior, según área de residencia y región natural, 2002-2011.

\begin{tabular}{lrrrrrrrrrr}
\hline $\begin{array}{l}\text { Área de residencia / } \\
\text { Región natural }\end{array}$ & 2002 & 2003 & 2004 & 2005 & 2006 & 2007 & 2008 & 2009 & 2010 & 2011 \\
\hline Total & 20,9 & 21,6 & 19,0 & 18,5 & 21,2 & 23,0 & 24,3 & 25,6 & 26,2 & 27,3 \\
$\quad$ Urbana & 26,1 & 27,7 & 23,8 & 23,1 & 26,6 & 28,2 & 29,8 & 30,9 & 31,1 & 31,9 \\
$\quad$ Rural & 8,2 & 7,2 & 6,9 & 6,8 & 6,8 & 8,9 & 8,6 & 10,3 & 10,8 & 12,4 \\
Costa & 23,3 & 24,0 & 21,5 & 20,5 & 24,2 & 26,1 & 27,0 & 28,8 & 28,0 & 29,6 \\
$\quad$ Sierra & 20,9 & 21,8 & 18,3 & 18,2 & 20,3 & 21,6 & 23,2 & 24,2 & 26,7 & 26,9 \\
$\quad$ Selva & 11,0 & 12,6 & 10,7 & 10,9 & 11,5 & 14,5 & 15,8 & 16,5 & 17,4 & 18,4 \\
\hline
\end{tabular}

Fuente: Instituto Nacional de Estadística e Informática - Encuesta Nacional de Hogares.

\section{Las perspectivas futuras de la cobertura}

$\mathrm{Si}$ se mantienen las tendencias podemos suponer un incremento anual del $2 \%$ de la cobertura promedio en el período de los próximos 18 años con un escenario al 2025. Ello permitiría alcanzar una tasa de cobertura de $70 \%$ sobre la población de 20 a 24 años. Del $31 \%$ actual pasaríamos al $70 \%$ en el 2025.

En relación con la población estudiantil, de los 15,7 millones al 2005 un incremento promedio del $10 \%$ permitiría alcanzar a una población estudiantil de 38 millones. A su vez, manteniendo las tendencias de expansión de la educación pública asociadas al crecimiento del PBI como ha venido ocurriendo, el sector público pasaría de 8 millones de estudiantes terciarios actuales a 12 millones, y el sector privado de 7,5 millones en el 2006 a 26 millones en el 2025, con lo que el sector privado pasaría del $48 \%$ de la matrícula actual al $68 \%$, en tanto que el sector público se reduciría del $52 \%$ actual al $32 \%$ de cobertura de la matrícula terciaria (5).

\section{Determinantes de la expansión privada}

- Oferta de carreras de menor duración

- Diferenciación institucional y geográfica

- Ofertas más adaptadas a las demandas del mercado

- Procedimientos administrativos más ágiles

- Menores requisitos de ingreso
- Menores o mayores niveles de calidad

- Sistemas de créditos y becas

- Afinidad de valores a los estudiantes

- Estabilidad institucional y administrativa

- Disminución del acceso a la educación pública gratuita

- Mejor empleabilidad

\section{Nuevas tendencias de la calidad}

- Acreditación por carreras versus la acreditación institucional

- Voluntariedad versus la obligación

- Evaluación cualitativa versus la cuantitativa

- Evaluación por resultados a través de exámenes

- Evaluación en función de los principios y misiones versus parámetros de calidad predefinidos

- Preeminencia de la evaluación externa versus la autoevaluación

- Preeminencia de los sistemas informáticos frente a las evaluaciones presenciales

\section{Megatendencias que transformarán el sector de la educación superior}

\section{- Democratización del aprendizaje y} acceso

Tradicionalmente, las universidades eran la clave de conocimiento, tanto en un sentido físico y filosófico, las bibliotecas de la universidad e institutos de investigación son el lugar donde el conocimiento se crea, almacena y 
comparte. Hoy en día el conocimiento está abierto a cualquier persona a nivel mundial con un dispositivo y la conectividad. El aumento masivo de la disponibilidad de "conocimiento" en línea y la expansión masiva del acceso a la enseñanza universitaria en los mercados desarrollados y en desarrollo supondrá un cambio fundamental en el papel de las universidades como creadores y guardianes del conocimiento (6).

- Competencia en los mercados y en el financiamiento

La competencia en los mercados y en el financiamiento se profundizará tanto a nivel nacional como en el plano internacional, con el crecimiento de financiamiento procedente de fuentes altamente competitivas y recursos no gubernamentales.

\section{- Tecnologías digitales}

Las tecnologías digitales transformarán radicalmente la forma de crear valor en la educación superior y las industrias relacionadas. Las tecnologías digitales y la innovación han irrumpido todo tipo de industrias establecidas, los medios de comunicación, comercio, entretenimiento y muchos otros. (6). Estas tecnologías, sin embargo, no causarán la desaparición de la universidad basada en el campus, por lo que esta seguirá existiendo como lugar de enseñanza, aprendizaje, investigación, participación comunitaria y para las diferentes experiencias de los estudiantes.

\section{- Movilidad global}

El mercado internacional estudiantil está creciendo rápidamente (un crecimiento global del $6,6 \%$ anual en la última década), pero cambiará fundamentalmente en estructura durante la próxima década y en lo sucesivo, ya que los mercados emisores tradicionales como China, Malasia, Corea del Sur y otros, se convierten cada vez más en destinos de escala mundial para los estudiantes internacionales (6).
Asimismo, las fuentes de talento académico serán más difusas debido a que los mercados emergentes son cada vez más móviles en su demanda, proporcionando una fuente creciente de talento para las universidades en las economías desarrolladas como en vías de desarrollo. Es muy probable que en los próximos 10 a 15 años surja un número pequeño de "marcas" de universidades de élite, verdaderamente global (6). Estas marcas incluirán algunas instituciones de élite de China ya que cuentan con los recursos, el apoyo gubernamental y la intención de alcanzar el estatus de élite mundial.

\section{- Integración con la industria}

La relación entre la industria y el sector de educación superior está cambiando y profundizándose. La industria desempeña múltiples funciones como cliente y socio de las instituciones de educación superior y cada vez más, como competidor (6).

Para que las universidades sobrevivan y prosperen, tendrán que establecer relaciones mucho más estrechas con la industria en la próxima década. La escala y profundidad de aprendizaje de la industria será cada vez más importante como fuente de ventaja competitiva para aquellas universidades que tienen asociaciones con la industria y pedagogía.

Por último, la industria va a competir cada vez más con las universidades en una serie de programas profesionales especializados.

\section{Impulsores del cambio}

Estos impulsores del cambio van a transformar el panorama de la educación superior, por lo que obliga a las universidades a adaptar sus modelos de negocio. La suma total de estos factores de cambio generará un panorama de un mercado de educación superior muy diferente dentro de 15 años (6). 


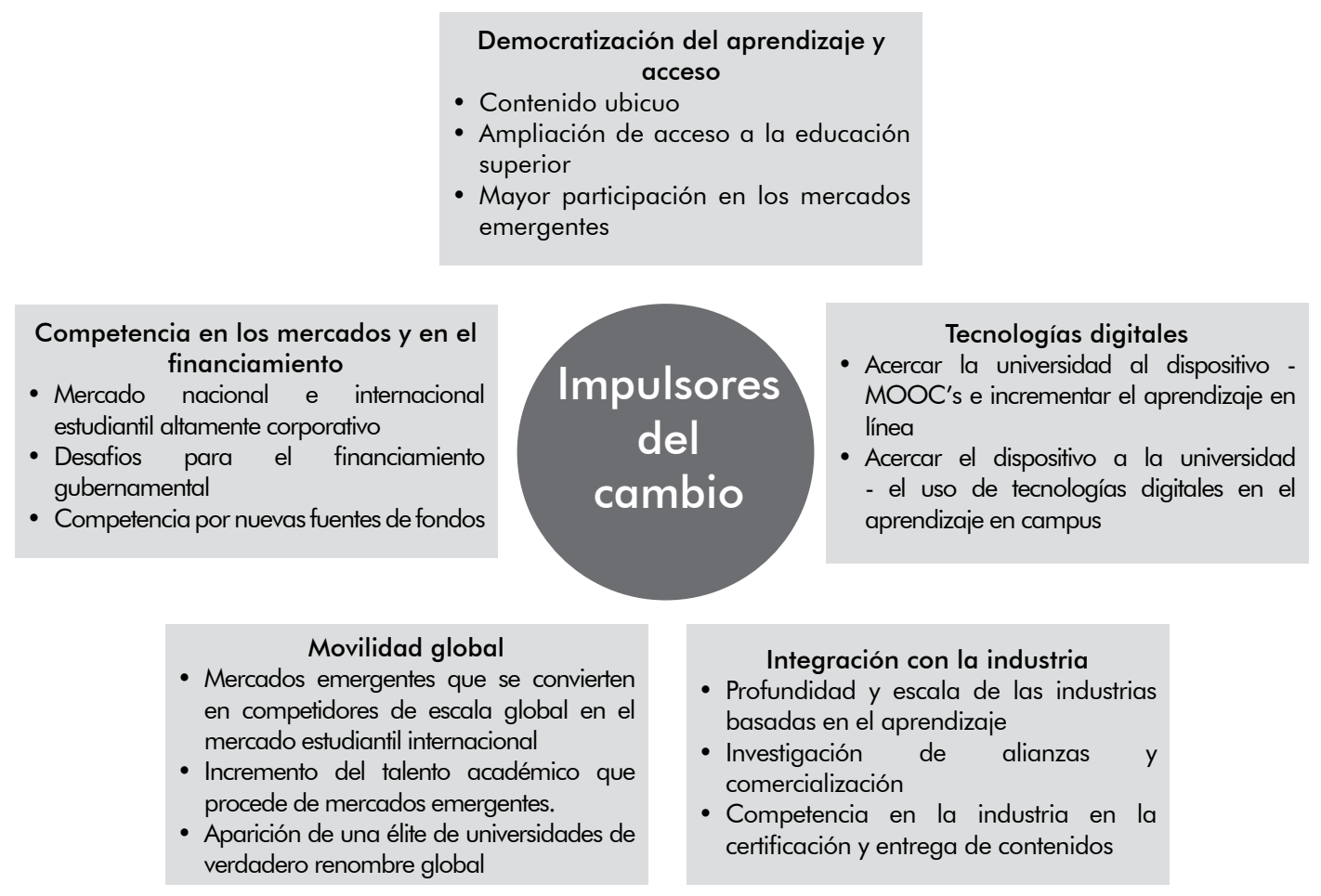

Figura №3: Megatendencias que transformarán el sector de la educación superior.

\section{Visión del sector de desarrollo}

- Las universidades se verán obligadas a crear nuevos modelos de negocio menos densos y dinámicos en la medida en que aumente la competencia de personal, estudiantes, financiación y socios.

- Las instituciones públicas se verán cada vez más funcionando como empresas, incrementando su esfuerzo de mantener la libertad de investigación y el rigor académico de las que su reputación depende a largo plazo.

- Las instituciones privadas explotarán nichos de mercado rentables, mientras que otros van a crear nuevos mercados y fuentes de valor, por ejemplo, mediante la especialización en partes selectas de la cadena de valor de la educación.

- Los responsables políticos tratarán de mantener un crecimiento constante en el acceso a la educación universitaria. Ellos buscarán palancas y programas que ubiquen al sector de educación superior en el centro de una economía de conocimiento genuino integrado a la región de Asia, mientras que inevitablemente se presionará por cada vez una mayor calidad de proveedores.

\section{Cambios que forzarán a las universidades a adaptarse de diferentes maneras}

- Ampliación de los programas: Las universidades tendrán que considerar que sí pueden seguir manteniendo una posición competitiva nacional e internacional a través de una amplia gama de programas.

- Cliente como target: Tener una estrategia clara alrededor de diferentes segmentos de alumnos, en función de sus necesidades y preferencias específicas. Hoy la segmentación de la mayoría de las universidades es amplia y las que no se enfoquen más en los segmentos estarán expuestas ante competidores con mejores propuestas estudiantiles.

- Canales de mercado: Las universidades 
tendrán que asumir el papel de los canales digitales y sociedades con terceros en el reclutamiento de alumnos, así como en la entrega de programas de enseñanza e investigación.

- Back office: La base de activos y administración de la universidad tendrá que ser mucho menos pesada de lo que es hoy. La mayoría de las universidades en la actualidad cuentan con mayor cantidad de personal de apoyo que personal académico, esta relación tendrá que cambiar.

\section{Evolución del modelo de la universidad}

El modelo de universidad dominante en el mundo es una base amplia para la enseñanza e investigación, soportado en una amplia base de activos y en su mayoría de back office in house.

\section{- Statu quo simplificado}

Algunas universidades establecidas que siguen funcionando como la enseñanza de base amplia y las instituciones de investigación que van a transformar progresivamente la forma en que prestan sus servicios y administran sus organizaciones, con implicaciones importantes para la forma en que se involucran con los estudiantes, gobierno, representantes de la industria, las escuelas secundarias y la comunidad.

\section{- Dominación de nichos}

Algunas universidades rediseñarán y redefinirán los servicios y mercados en los que operan, cambiando su modelo de negocio, forma de organización y operaciones.

\section{- Transformers}

Los proveedores privados y los nuevos participantes tendrán que forjar nuevas posiciones en el sector tradicional, creando nuevos mercados que unan la educación superior con otros sectores.

Los modelos descritos presentan tres líneas de evolución del negocio de la universidad.
Hay, por supuesto, otros modelos potenciales, incluyendo: los modelos de aprendizaje para toda la vida, los modelos globales de alianzas, los modelos multidisciplinarios, y los modelos híbridos.

\section{¿Cómo construir una universidad innovadora?}

Hacer de la universidad una organización innovadora no es muy distinto que hacerlo de cualquier otra organización, pero lógicamente tiene sus singularidades. Más allá que de algunas universidades hayan salido grandes propuestas de innovación y spin-off de referencia, lo cual no es menor, las universidades en general no son organizaciones que destaquen por aplicarse la innovación a sí mismas, son más bien proclives a recetar la innovación a los demás y excusarse en la púrpura académica (7).

Once aspectos clave para hacer de la universidad una organización innovadora:

- Voluntad de cambiar para no perder de vista el cambio externo, para poder continuar ofreciendo valor a estudiantes, a empresas, a la sociedad en general.

- Definir retos estratégicos clave que pueden ser más fácilmente alcanzados desde la innovación que desde la planificación estratégica.

- Apostar por una cultura innovadora, capaz de cuestionar ortodoxias, de abrazar la lateralidad, de arriesgar emprendiendo nuevas soluciones.

- Definir un modelo de innovación que permita sistematizar la innovación como algo continuo, no como algo esporádico y periférico.

- Dedicar talento a la innovación como institución, crear equipos de innovación con capacidad de aunar creatividad y disciplina de cambio. En las universidades se generan muchas ideas pero el ratio de innovación es muy bajo. 


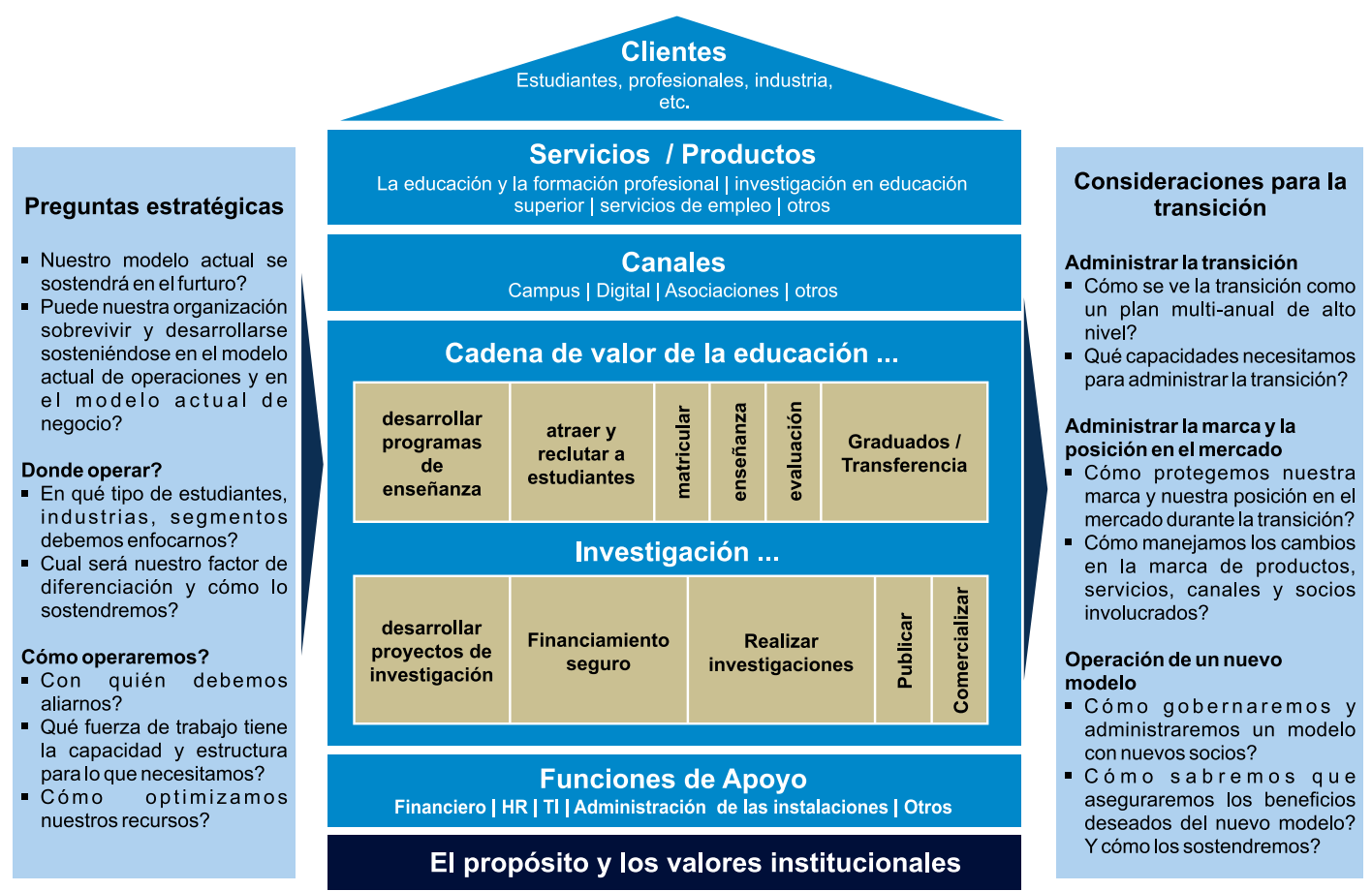

Figura $N^{\circ}$ 4: Marco para la evaluación y el diseño de un modelo de universidad para el futuro propuesto por Ernst \& Young's.

- Estar atentos a los riesgos de disrupción, no todo el mundo puede ser Harvard y fenómenos como los MOOC.

- Establecer una verdadera vigilancia tecnológica que permita ayudar a aprender de otros modos, usando nuevas palancas, como las redes sociales.

- Aprovechar los beneficios de combinar talento interno y talento externo a la hora de innovar desde una clara apuesta por la innovación abierta.

- Explorar nuevos modelos de negocio, los actuales serán cuestionados y se ven como claramente insuficientes en muchos sistemas universitarios.

- Definir un nuevo paradigma de tech transfer, la vieja ecuación universidad empresa hace aguas desde que se rompió la linealidad I+D+I (investigación, desarrollo e innovación).

- Establecer una gobernanza que se aleje del corporativismo y sea capaz de asumir la innovación y el cambio sin traumatismos infinitos.

\section{CONCLUSIONES}

En América Latina y el Caribe se han presentado a partir del siglo $\mathrm{XX}$, tres reformas de la educación superior. En la tercera, sufren un verdadero shock. Hoy estos sistemas de educación superior han internacionalizado las nuevas tecnologías de comunicación e información, ya que las demandas de acceso de la población y la presencia creciente de las sociedades del conocimiento lo requieren.

Las universidades deberían establecer, si su modelo actual es a prueba del futuro, $y$, si no, determinar dónde y cómo enfrentar el futuro, teniendo en cuenta estratégicas relacionadas con la viabilidad de su modelo actual. Las deliberaciones sobre la futura necesidad de incluir modelos de segmentos enfocados en los servicios que necesitan los clientes y los canales de comercialización cumplen su papel dentro de la cadena de valor de las universidades. Las funciones de la ayuda también necesitarán ser aerodinamizadas. 
Independientemente del modelo y la dirección elegida, los retos que las universidades tendrán que superar, a fin de que puedan implementar nuevos modelos, las consideraciones clave para los encargados es formular políticas, y se dan las oportunidades para las universidades del sector privado. Las implicaciones para el sector privado debido a su valor, deben ser más directas: encontrar oportunidades para crear y diseñar los modelos de negocio necesarios para concretarlos. Los mercados de educación superior tienen excelentes fundamentos para un crecimiento a largo plazo y proporcionar acceso a la creciente clase media en los mercados emergentes.

\section{REFERENCIAS BIBLIOGRÁFICAS}

1. Toffler A. El shock del futuro. 8 8 $^{\mathbf{a}}$ ed. Barcelona: Plaza \& Janes Editories S.A.; 1995.

2. Rama C. La tercera reforma de la educación superior en América Latina y el Caribe. $1^{\underline{a}}$ ed. Buenos Aires: Fondo de Cultura Económica USA; 2006.

3. Rama C. Las nuevas tendencias de la Educación Superior en América Latina. Lima: Asamblea Nacional de Rectores; 2008.

4. Instituto Nacional de Estadística e Informática. Indicadores de Educación por Departamentos: 2001 - 2011. Perú.

5. Rama C. Estudio de tendencias de educación superior en América Latina. Asamblea Nacional de Rectores, Perú.

6. Okor J. University of the future, Australia: Ernst \& Young; 2012.

7. Christensen $\mathrm{C}$, Eyring $\mathrm{H}$. The Innovative University: Changing the DNA of Higher Education from the Inside Out. la ed. New York, USA: John Wiley \& Sons; 2011. 